\title{
The role of ultrasound examination in the management of a patient with hemoperitoneum and an ovarian mass: a clinical and diagnostic challenge
}

\section{To the Editor,}

Ovarian metastasis is a rare presentation of endometrial cancer. Moreover, it is a sporadic cause of hemoperitoneum causing severe anaemia (1). Therefore we decided to describe this case of hemoperitoneum resulting in severe anaemia associated with an ovarian mass which was diagnosed as metastasis of endometrial cancer. The aim of this report was to highlight some observations about this case.

We report the case of a 49-year-old, nulliparous woman with abdominal swelling and severe abdominal pain. She presented with fever and tachycardia. Blood tests showed severe anaemia (hemoglobin: $3.8 \mathrm{~g} / \mathrm{dL}$ ). Both transvaginal and transabdominal ultrasound examinations were performed. They showed thickened, vascularized endometrium of $18 \mathrm{~mm}$, irregular myometrial-endometrial junctions, and a large solid tumor in the right side of the pelvis, measuring $100 \mathrm{~mm}$ at the largest diameter with a regular external wall. Color Doppler examination indicated that the tumor was richly vascularized. The left ovary appeared normal. Both ascites and free fluid in the pouch of Douglas were also noted, suggesting hemoperitoneum. Immediately after hemodynamic stabilization and blood transfusions the patient underwent surgery. Laparotomy confirmed the presence of hemoperitoneum and of a large right ovarian mass. The right ovarian mass was removed and the frozen section was positive for borderline tumor (Figure 1). Considering the age of the patient and the ultrasound findings, a radical hysterectomy (Morrow \& Querleu type A) and bilateral salpingo-oophorectomy were performed. Final histology reported grade 3 endometrioid carcinoma of the endometrium with $88 \%$ myometrial invasion; vagina and parametria were infiltrated. The ovarian mass was found to be a metastasis from the endometrial cancer. The left ovary was described as normal to histopathological examination. The patient underwent chemotherapy, radiotherapy and brachytherapy with good clinical response; she was disease free at 28 months follow-up. This case was notable for the following peculiarities. Hemoperitoneum and severe anaemia are rarely observed in patients with endometrial cancer. Endometrial cancer usually presents with abnormal uterine bleeding, but this was not the manner of presentation in this patient. Ovarian metastasis is also a rare finding in endometrial cancer $(2,3)$ and it is usually bilateral (4), whereas in the present case the metastasis was unilateral. Our results agree with those previously reported by other authors: ovarian metastases from endometrial cancer usually appear as vascularized solid tumor (4).

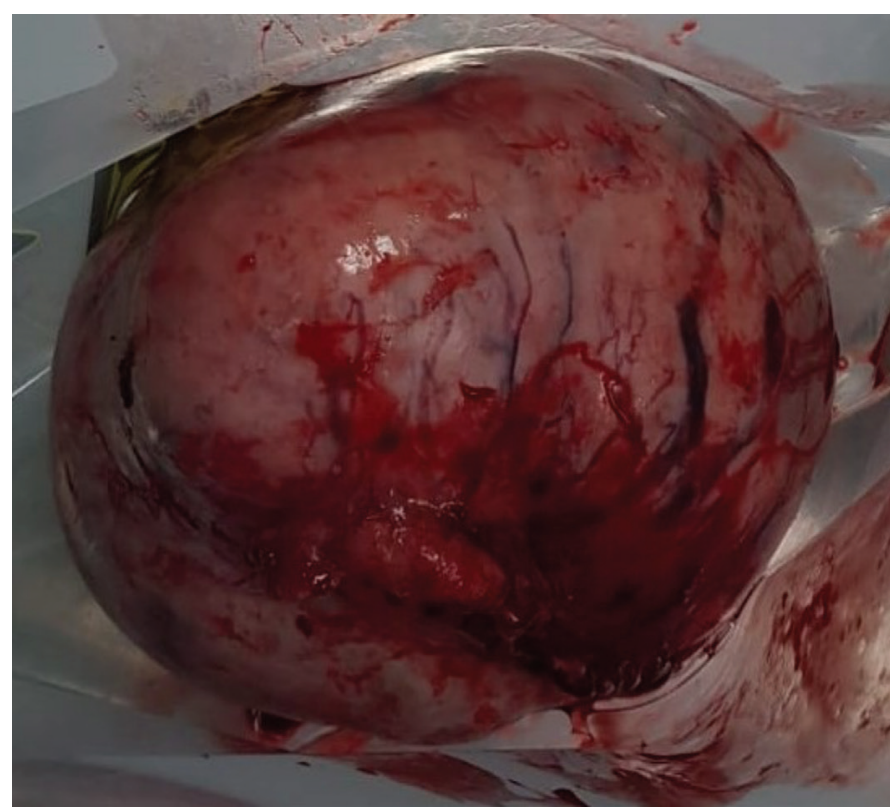

Figure 1. Ovarian mass, send for preliminary histological examination 
This case highlights the difficulties in diagnosing endometrial cancer in the absence of typical symptoms and risk factors. It also emphasizes the key role of ultrasound examination in an emergency setting, in this instance providing guidance to the surgeon to enable planning of the best surgical treatment.

Finally, it should be remembered that only the final histology report will provide the definitive and correct final diagnosis. In our patient, a borderline tumor was suspected at the time of the frozen section, but final histology was positive for an invasive tumor. Borderline tumors require different management from invasive tumors (5). In this case the surgeon performed the correct surgery immediately, because the ultrasound findings suggested a malignant tumor.

We present a patient with endometrial cancer which had metastasized to the ovary and that was discovered because of the unusual presentation of hemoperitoneum and severe anaemia. In addition, the ultrasound examination played an important role in the timely management of this patient.

Maria Donata Spazzini, Laura Carlini, Paola Algeri, Santina Ermito, Massimo Ciammella

Department of Obstetrics and Gynecology, Bolognini Hospital, ASST-Bergamo Est, Bergamo, Italy

\section{References}

1. Braun MM, Overbeek-Wager EA, Grumbo RJ. Diagnosis and management of endometrial cancer. Am Fam Physician 2016; 93: 468-74.

2. Dogan A, Schultheis B, Rezniczek GA, Hilal Z, Cetin C, Häusler G, et al. Synchronous endometrial and ovarian cancer in young women: case report and review of the literature. Anticancer Res 2017; 37 : 969-78.

3. Ryan M, Laios A, Pathak D, Weston M, Hutson R. An unusual presentation of endometrial cancer with bilateral adrenal metastases at the time of presentation and an updated descriptive literature review. Case Rep Obstet Gynecol 2019; 2019: 3515869.

4. Moro F, Leombroni M, Pasciuto T, Trivellizzi IN, Mascilini F, Ciccarone F, et al. Synchronous primary cancers of endometrium and ovary vs endometrial cancer with ovarian metastasis: an observational study. Ultrasound Obstet Gynecol 2019; 53: 827-35.

5. Gershenson DM. Management of borderline ovarian tumours. Best Pract Res Clin Obstet Gynaecol 2017; 41: 49-59. 